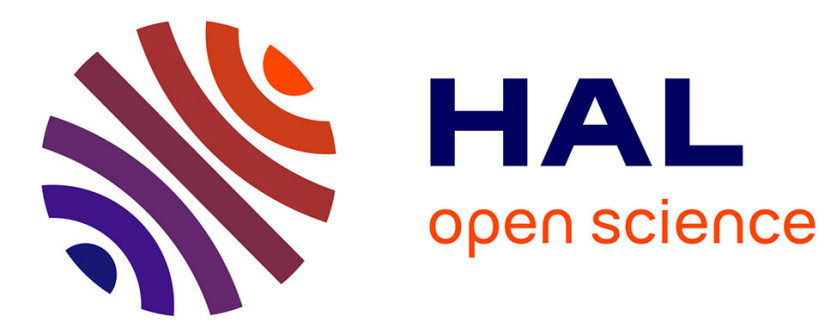

\title{
An Isotropization Mechanism in Bianchi I Model
}

Henri-Hugues Fliche, Roland Triay, Màrio Novello, Luciane R. de Freitas

\section{To cite this version:}

Henri-Hugues Fliche, Roland Triay, Màrio Novello, Luciane R. de Freitas. An Isotropization Mechanism in Bianchi I Model. International Journal of Modern Physics D, 2003, 12, 8, pp.1499. 10.1142/S0218271803003682 . hal-00005060

\section{HAL Id: hal-00005060 https://hal.science/hal-00005060}

Submitted on 1 Jun 2005

HAL is a multi-disciplinary open access archive for the deposit and dissemination of scientific research documents, whether they are published or not. The documents may come from teaching and research institutions in France or abroad, or from public or private research centers.
L'archive ouverte pluridisciplinaire $\mathbf{H A L}$, est destinée au dépôt et à la diffusion de documents scientifiques de niveau recherche, publiés ou non, émanant des établissements d'enseignement et de recherche français ou étrangers, des laboratoires publics ou privés. 


\title{
AN ISOTROPIZATION MECHANISM IN BIANCHI I MODEL
}

\author{
H.H. FLICHE
}

LMMT, UPRES EA 2596, Fac. des Sciences et Techniques de St Jérôme, av. Normandie-Niemen 13397 Marseille Cedex 20, France*

\section{R. TRIAY}

Université de Provence and Centre de Physique Théorique-CNRS, UP 7061, Luminy Case 907 F 13288 Marseille Cedex 9, France $^{\dagger}$

\section{NOVELLO}

Centro Brasileiro de Pesquisas Físicas, Rua Dr. Xavier Sigaud, 150, Urca 22290-180 Rio de Janeiro RJ, Brazil

L.R. de FREITAS

Departamento de Física Matemática, Instituto de Física, UFRJ, Cidade Universitária CEP:21945-180, Rio de Janeiro RJ, Brazil ${ }^{\S}$

We ask whether the problem on the initial conditions in Cosmology can be solved if an efficient elimination mechanism of the anisotropy was present at earlier epochs. We explore the consequences of the existence of an epoch in which the material content of the Universe was represented by an stress-energy tensor possessing anisotropic pressure which depends non linearly on the expansion parameter. We show that for candidate behaviors the associated shear pass through a maximum and vanishes asymptotically. We use the Hamiltonian formalism to define a constant of motion which enables us to classify the cosmological world-models.

Keywords: Cosmology, Primordial era

\section{Introduction}

Among fundamental questions on Cosmology, we may ask why the standard model is conformally flat, the conformal factor depends solely on the cosmological time, and what is the issue of the Horizon problem. In other words, what is the origin such a peculiar symmetry of geometrical properties of the universe, when a non vanishing Weil tensor does not preserve homogeneity and the existence of conformal Killing vectors? In particular, the presence of shear in the cosmological fluid is difficult to avoid, even for weak perturbations of FRW

\footnotetext{
*henri-hugues.fliche@univ.u-3mrs.fr

†triay@cpt.univ-mrs.fr

${ }_{\ddagger}$ novelloy@lafex.cbpf.br

$\S$ luciane@if.ufrj.br
} 
geometry. Instead of assuming ab nihilo the existence of an inflation period, we envisage another issue that is motivated by a non trivial observational fact. the blackbody spectrum of the cosmological microwave background radiation 9 (CMB). Such a feature shows that the cosmological medium is in a thermodynamical equilibrium, a property that suffices for answering partly the question 10 . In general, such a symmetry is acquired by means of dissipative process as the final state of a thermodynamical process, according to the second thermodynamics principle. For our purpose, we may safely assume that the early Universe was composed of a chaotic mixture of various species of elementary particles and radiation, and that dissipative processes were indeed present. In such a media, viscous processes (e.g. due to the neutrino-matter interactions, 5 ) could have contributed to the isotropization, although it has been shown that such a result is not as easily ensured as expected 11. 6 . Since the physics of the early universe is not known, we turn this question on the way around by proposing candidate dynamics (toy-model) whichevolves asymptotically to Friedmann models. Sim-

ilarly to other investigation 3 , we expect that generic behaviors can be classified by means of geometrical criteria (e.g., the isotropization due to a cosmological constant 11 ).

Herein, we present a new analysis of Misner hypothesis which has been proposed three decades ago. Namely, one may ask whether a primordial shear $\Sigma$ can be cancelled with the expansion by the presence of viscous processes. Instead of a linear relationship between the anisotropic pressure and the shear, which does not account for an effective isotropization mechanism, we extend Misner approach by assuming a viscous process which depends on the expansion factor $\Theta$ by means of a second degree polynomial. The isotropization mechanism is investigated within a Bianchi I geometry.

\section{The Material Content of the Universe}

Let us assume the existence of a global gaussian system of coordinates, which enable us to define the cosmological time $t$. Let $V^{\mu}$ be the four-velocity of the cosmological fluid, $V^{\mu} V_{\mu}=1$. It is worth to decompose its derivative in terms of its irreducible components : the symmetric traceless shear tensor $\sigma_{\mu \nu}$ and the Hubble parameter $\Theta=V_{; \mu}^{\mu}$ (volume expansion) once there is no vorticity. By using the projector $h_{\mu \nu}$ on the 3-dimensional rest-space of the commoving observer by

$$
h_{\mu \nu}=g_{\mu \nu}-V_{\mu} V_{\nu}
$$

the shear tensor is defined in the usual way

$$
\sigma^{\alpha \beta}=\frac{1}{2} h^{\mu \alpha} h^{\beta \lambda} V_{(\mu ; \lambda)}-\frac{1}{3} \Theta h^{\alpha \beta}
$$

and the covariant derivative of $V_{\mu}$ reads

$$
V_{\mu ; \nu}=\sigma_{\mu \nu}+\frac{1}{3} \Theta h_{\mu \nu}
$$

The stress-energy tensor of the cosmological fluid is defined by

$$
T_{\mu \nu}=\rho V_{\mu} V_{\nu}-p h_{\mu \nu}+\Pi_{\mu \nu}
$$

where $\rho$ is the energy density of gravitational sources, $p$ the isotropic pressure and $\Pi_{\mu \nu}$ is the traceless anisotropic pressure tensor. Hence, Einstein equations provide us with Raychaudhuri's equation for the expansion factor

$$
\dot{\Theta}+\frac{1}{3} \Theta^{2}+\frac{1}{2}(\rho+3 p+3 \Pi)=0
$$


and the evolution equation for the shear tensor

$$
h_{\alpha}^{\mu} h_{\beta}^{\nu} \dot{\sigma}_{\mu \nu}-\frac{1}{3}\left(\omega^{2}+2 \sigma^{2}\right)+\frac{2}{3} \Theta \sigma_{\alpha \beta}+\sigma_{\alpha}^{\mu} \sigma_{\mu \beta}=R_{\alpha \epsilon \beta \nu} V^{\epsilon} V^{\nu}-\frac{1}{3} R_{\mu \nu} V^{\mu} V^{\nu} h_{\alpha \beta}
$$

where

$$
\sigma^{2}=\frac{1}{2} \sigma_{\mu \nu} \sigma^{\mu \nu}
$$

We extend Misner approach by assuming

$$
\Pi_{\mu \nu}=\left(\alpha_{0}+\alpha_{1} \Theta+\alpha_{2} \Theta^{2}\right) \sigma_{\mu \nu}
$$

where $\alpha_{i=0,1,2}$ are constants which characterize the physical process. Within such an assumption, because $\Pi_{\mu \nu}$ depends on $\sigma_{\mu \nu}$ and quadratically on $\Theta$, a phase transition can occurred in the evolution of the cosmological fluids 6 . 6 .

We assume a cosmological fluid with a planar anisotropy, the shear tensor reads

$$
\sigma_{i}^{j}=\Sigma\left(\begin{array}{ccc}
2 & 0 & 0 \\
0 & -1 & 0 \\
0 & 0 & -1
\end{array}\right)
$$

and with an equation of state defined by

$$
p=(\gamma-1) \rho, \quad 0 \leq \gamma \leq 2
$$

where $\gamma$ accounts for the state of matter (e.g., $\gamma=\frac{4}{3}$ for radiation, $\gamma=1$ for dust like matter). Written in terms of two variables $\Theta$ and $\Sigma$, Eq. (5.6) transform

$$
\begin{aligned}
& \dot{\Theta}=-\tilde{\gamma} \Theta^{2}-9(1-\tilde{\gamma}) \Sigma^{2}, \quad \tilde{\gamma}=\frac{\gamma}{2} \\
& \dot{\Sigma}=f(\Theta) \Sigma
\end{aligned}
$$

where the polynomial

$$
f(\Theta)=-\left(\alpha_{0}+\left(1-\alpha_{1}\right) \Theta+\alpha_{2} \Theta^{2}\right)
$$

This equations system provides us with dynamics for the investigation of the isotropization process in term of parameters $\alpha_{i=0,1,2}$. According to Eq. (11), the cosmological expansion decelerates

$$
\dot{\Theta} \leq-\tilde{\gamma} \Theta^{2}
$$

since the origin of time and the larger the magnitude of the shear the slower the expansion rate. It decouples from the shear if $\gamma=2$ (ultra-relativistic Zeldovich fluid); and in such a case, a obvious calculation provides us with

$$
\Sigma \propto \exp \left(-\frac{\alpha_{0}}{\Theta}+\left(1-\alpha_{1}\right) \ln \Theta+\alpha_{2} \Theta\right)
$$

Otherwise, since $(\dot{\Theta}<0)$ the expansion factor $\Theta$ reads as a decreasing monotonic function in terms of the cosmological time $t$, the function $t \mapsto \Theta(t)$ is a one to one mapping. Therefore $\Theta$ can be used as a time parameter, and we write

$$
t=t(\Theta), \quad \Sigma=\Sigma(\Theta)
$$




\section{3. isotropization mechanism}

The aim of this section is to investigate the extended Misner isotropization process in term of parameters $\alpha_{i=0,1,2}$ in a Bianchi I cosmology. We limit the investigation to candidate behaviors which lead to isotropy when the universe undergoes its cosmological expansion. According to Eq. (12,13), the condition

$$
\alpha_{0} \geq 0
$$

is required for a vanishing shear at the end of the isotropization process. The evolution of the anisotropy is investigated by means of variables $\Theta$ and $\Sigma$, which are governed by an autonomous differential equations system as given by Eq. (11, 12). The application of the theory of dynamical systems enables us to characterize the solutions by a control parameter $H$ (constant of motion), see Sec. (). It acts as the only meaningful order parameter that specifies the dynamics of the expansion (indeed, initial conditions of $\Theta$ and $\Sigma$ cannot be not defined at Big Bang). It turns out that exact solutions can be derived, see Sect. . They correspond to generic trends which help us to classify more complex behaviors. Instead of $\Sigma$, the dimensionless quantity

$$
\zeta=\frac{\Sigma^{2}}{\Theta^{2}}
$$

hereafter called shear rate, is more adapted to characterize the isotropization process with the condition

$$
\zeta \rightarrow 0
$$

It turns out that the dynamics can be defined in terms of dimensionless variables, see Sect. . Such a description enables us to investigate isotropization processes regardless of chronology. Hence, any candidate process can be rescaled to observational and/or physical constraints. The stability of the isotropization mechanism is investigated about the background shear-free dynamics, see Sec. (). Finally, a qualitative analysis of candidate processes is given in Sec. ().

\subsection{Control parameter}

Equations system defined in Eq. (11, 12) can be understood as the characteristic system of a first order partial differential equations system which reads

$$
\dot{\Theta}=\mu \frac{\partial H}{\partial \Sigma}, \quad \dot{\Sigma}=-\mu \frac{\partial H}{\partial \Theta}
$$

where $\mu=\mu(\Theta, \Sigma)$ is an integrating factor. The solutions are characterized by a constant of motion $H$, which satisfies the condition

$$
\frac{\mathrm{d} H}{\mathrm{~d} t}=\frac{\partial H}{\partial \Theta} \dot{\Theta}+\frac{\partial H}{\partial \Sigma} \dot{\Sigma}=0
$$

According to Eq. (11, 12, 13), Eq. (21) transforms

$$
\frac{\partial H}{\partial \Theta}\left(\tilde{\gamma} \Theta^{2}+9(1-\tilde{\gamma}) \Sigma^{2}\right)+\frac{\partial H}{\partial \Sigma}\left(\alpha_{0}+\left(1-\alpha_{1}\right) \Theta+\alpha_{2} \Theta^{2}\right) \Sigma=0
$$

Thanks to Eq. (16) nothing prevents us to write

$$
\Sigma^{2}=g(\Theta, H)
$$


where $g(\Theta, H)$ is a solution of equation

$$
\left(\tilde{\gamma} \Theta^{2}+9(1-\tilde{\gamma}) g(\Theta, H)\right) g^{\prime}(\Theta, H)+2 f(\Theta) g(\Theta, H)=0, \quad g^{\prime}=\frac{\partial g}{\partial \Theta}
$$

\subsection{Polynomial solutions}

If one assumes that $g(\Theta, H)$ reads as a polynomial in term of $\Theta$ then

$$
g(\Theta, H)=H \Theta^{n}, \quad n=1,2,3
$$

are the only solutions of Eq. (24), they are specified solely by $H$ and $\tilde{\gamma}$.

- if $n=1$ then $\zeta=H / \Theta$ and the dynamics is characterized by

$$
\alpha_{0}=\frac{9}{2}(1-\tilde{\gamma}) H, \quad \alpha_{1}=1-\frac{\tilde{\gamma}}{2}, \quad \alpha_{2}=0
$$

The chronology is given by

$$
t=\frac{1}{9 H(1-\tilde{\gamma})} \ln \left(1+\frac{9 H(1-\tilde{\gamma})}{\tilde{\gamma} \Theta}\right)
$$

The shear $\Sigma \propto \sqrt{\Theta}$ vanishes and $\Theta$ behaves asymptotically as in the free shear case but the isotropization process cannot be achieved.

- if $n=2$ then $\zeta=H$ and

$$
\begin{gathered}
\alpha_{0}=0, \quad \alpha_{1}=1-\tilde{\gamma}-9(1-\tilde{\gamma}) H, \quad \alpha_{2}=0 \\
t=\frac{1}{(\tilde{\gamma}+9 H(1-\tilde{\gamma})) \Theta}
\end{gathered}
$$

Similarly as above, the isotropization process cannot be achieved.

- if $n=3$ then $\zeta=H \Theta$ and

$$
\begin{gathered}
\alpha_{0}=0, \quad \alpha_{1}=1-\frac{3}{2} \tilde{\gamma}, \quad \alpha_{2}=\frac{27}{2}(1-\tilde{\gamma}) H \\
t=\frac{1}{\tilde{\gamma} \Theta}-9 H \frac{1-\tilde{\gamma}}{\tilde{\gamma}^{2}} \ln \left(1+\frac{\tilde{\gamma}}{9 H(1-\tilde{\gamma}) \Theta}\right)
\end{gathered}
$$

The isotropization process $\left(\zeta \sim t^{-2}\right)$ can be achieved.

With the idea in mind that the general solution of Eq. (24) can be approached by a polynomial expansion, we understand that isotropization requires

$$
\alpha_{2} \neq 0
$$

\subsection{Dimensionless description}


According to previous sections, we assume $\alpha_{0}>0$ and $\alpha_{2} \neq 0$. The equations system Eq. (11,12) reads in terms of the following dimensionless quantities

$$
\tau=\alpha_{0} t, \quad \eta=\frac{\alpha_{0}}{\Theta}, \quad \beta=\alpha_{0} \alpha_{2}
$$

where $\alpha_{0}$ provides us with a time scale, as follows

$$
\begin{aligned}
& \frac{\mathrm{d} \eta}{\mathrm{d} \tau}=\tilde{\gamma}+9(1-\tilde{\gamma}) \zeta \\
& \frac{\mathrm{d} \zeta}{\mathrm{d} \tau}=-2 \zeta\left(1+\left(1-\tilde{\gamma}-\alpha_{1}\right) \frac{1}{\eta}+\frac{\beta}{\eta^{2}}\right)+18 \zeta^{2}(1-\tilde{\gamma}) \frac{1}{\eta}
\end{aligned}
$$

The solutions depend on $\alpha_{1}, \beta$ and a dimensionless constant of motion $h$, it is defined by rewriting Eq. (23) as follows

$$
\zeta=\phi(\eta, h)
$$

According to Eq. (34, 35), the evolution of shear rate is given by

$$
\frac{\mathrm{d} \zeta}{\mathrm{d} \eta}=-2 \zeta \frac{\left.1+\left(1-\tilde{\gamma}-\alpha_{1}-9(1-\tilde{\gamma}) \zeta\right)\right) \frac{1}{\eta}+\beta \frac{1}{\eta^{2}}}{\tilde{\gamma}+9(1-\tilde{\gamma}) \zeta}
$$

where $\eta$ increases with $t$.

\subsection{Stability}

It is clear that the stability of the shear-free dynamics, which is defined by

$$
\eta_{\circ}=\tilde{\gamma} \tau, \quad \zeta_{\circ}=0
$$

preserves the candidate behavior to move away from its trend toward isotropy The first order linearization of Eq. (34) 35) about this solution provides us with

$$
\begin{aligned}
\frac{\mathrm{d}(\delta \eta)}{\mathrm{d} \tau} & =9(1-\tilde{\gamma}) \delta \zeta \\
\frac{\mathrm{d}(\delta \zeta)}{\mathrm{d} \tau} & =-2\left(1+\left(1-\tilde{\gamma}-\alpha_{1}\right) \frac{1}{\eta_{\circ}}+\frac{\beta}{\eta_{\circ}^{2}}\right) \delta \zeta
\end{aligned}
$$

Hence, with Eq. (38), Eq. 40) integrates

$$
\delta \zeta=\delta \zeta_{(i)} \exp -2\left(\tau+\frac{\left(1-\tilde{\gamma}-\alpha_{1}\right)}{\tilde{\gamma}} \ln (\tau)-\frac{\beta}{\tilde{\gamma}^{2} \tau}\right)
$$

where $\delta \zeta_{(i)}=\delta \zeta_{(i)}(\delta h)$ accounts for a variation of the constant of motion $h$. The exponential decreasing trend with time ensures the asymptotical stability of the candidate behavior from a sufficiently large values of $\tau$. However, at earlier epoch the tendency of the solution to disentangle from the shear-free dynamics depends on the sign of $\beta$. Hence, we can infer the following properties :

- If $\beta<0$ then the shear rate increases slowly up to a maximum and hence it decreases to vanish for large values of $\tau$. 
- If $\beta>0$ then the shear rate cannot be weak at primordial era and the above linearization procedure cannot be applied.

Because the behavior related to $\beta<0$ case is stable, we expect the exact solution to show a similar property.

\subsection{Qualitative analysis}

A straightforward analysis of Eq. (37) provides us with the following isotropization criteria for the asymptotic behavior of the shear rate : if the ratio

$$
\frac{\zeta}{\eta} \leq B<\frac{1}{9(1-\tilde{\gamma})}
$$

is bounded by a given value $B$ during the primordial expansion then the second inequality in Eq. (42) ensures that $\zeta$ decreases exponentially down to a vanishing value. This condition on the primordial shear guaranties an asymptotic isotropization.

According to Eq. (37), the evolution of the shear rate $\zeta$ depends strongly on its primordial trend, which is either increasing $(\beta<0)$ or decreasing $(\beta>0)$ with time $\eta$. These two generic behaviors are displayed (a curve herein denoted $\Upsilon$ ) on Fig. 1 and Fig. 2 for candidate behaviors. They obey a simple rule that is based on whether $\Upsilon$ crosses or does not a curve $\Gamma$ (the extrema of $\zeta$ ) defined by

$$
\zeta=\frac{1}{9(1-\tilde{\gamma})}\left(1-\tilde{\gamma}-\alpha_{1}+\eta+\frac{\beta}{\eta}\right)
$$

This curve $\Gamma$ demarcates two value domains in the first quadrant $: \mathcal{D}^{+}$(where $\left.\frac{\mathrm{d} \zeta}{\mathrm{d} \eta}>0\right)$ and $\mathcal{D}^{-}\left(\right.$where $\left.\frac{\mathrm{d} \zeta}{\mathrm{d} \eta}<0\right)$. Let us proceed to the analysis of the isotropization process on these figures :

- If $\beta<0$ (see Fig. 1) then the shear rate increases with time at primordial era, $\Upsilon$ lies in $\mathcal{D}^{+}$(located on the left hand side of $\Gamma$ ). It reaches its maximum when $\Upsilon$ crosses $\Gamma$ and then it decreases with $\eta$ down to a vanishing value. These behaviors are similar to the ones related to linear perturbations.

- If $\beta>0$ (see Fig. 2) then $\Upsilon$ lies in $\mathcal{D}^{-}$at primordial era and the shear rate decreases with time. If $\Upsilon$ never crosses $\Gamma$ then the shear rate vanishes with time. Otherwise the condition which ensures isotropization is that $\Upsilon$ crosses $\Gamma$ twice.

\section{Isotropy and structures formation}

In the standard scenario it has been argued that causality requires the existence of an inflation period, although such a scenario can be questioned 12.13 . While it might be a sufficient condition for primordial non-homogeneities to disappear, our result shows that it is far from being necessary since isotropic asymptotical solutions exist according to specific criteria as described above. It is clear that such candidate behaviors have to be understood as ersatz regardless of physics. However, a realistic description of the physical process which could take place at primordial era has to verify large scale properties related to conditions described above. At small scales, one can expect phenomena which are typical of fluid mechanics ones, such as vortex creation. With this in mind, 


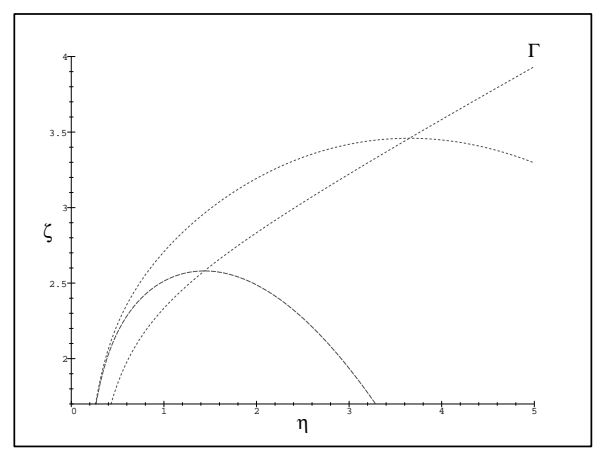

Figure 1: $\beta<0$

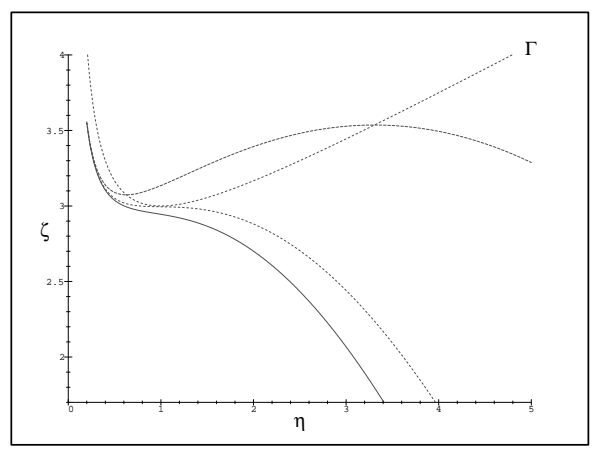

Figure 2: $\beta>0$ 
it is interesting to point out a peculiar behavior related to the $\beta>0$ case where the shear rate exhibits a phase transition like behavior by reaching a minimum and then a maximum, similarly as a shear burst (SB). Such a SB feature might account for a pure relativistic process which favors the primordial structures formation.

\section{Conclusion}

We present a possible isotropization process in Bianchi I cosmology based on Misner viscous process with an anisotropic pressure which is proportional to a second order polynomial of the expansion factor $\Theta$. The efficiency of this mechanism depends strongly on the highest term of the polynomial. It turns out that candidate behaviors admit a shear burst (SB) epoch which may play in the primordial structures formation. Hamiltonian formalism is preferred for classifying cosmological solutions regardless of the singularity problem. The dynamics is investigated by means of dimensionless quantities, such as the shear rate (the shear to expansion ratio), which enables us to rescale the isotropization mechanism to physical constraints.

6.

Acknowledgments M. Novello thanks Université de Provence and Centre de Physique Théorique for financial support.

\section{References}

1. V.A. Belinsky, I.M. Kalatnikov, JETP (1968) 151,431

2. V.A. Belinsky, I.M. Kalatnikov, JETP (1976) 42,205

3. S. Hawking, Mont. Not. Roy. Soc. (1996) 142,129

4. C.W. Misner, Phys. Rev. Lett. (1967) 19,533

5. C.W. Misner, Astrophys.J. (1968) 151,431

6. M. Novello, S.L.S. Duque, Physica A (1990) 168,1073

7. M. Novello, S.L.S. Duque, R. Triay, H.H. Fliche, Phys. Rev. D (1993) 47,3165

8. M. Novello, L.R. de Freitas, Int. J. Mod. Phys. A (1998) 13, 363

9. G.F. Smooth etal., Astro. Phys. J. (1992) 396,L1

10. J.M. Souriau, Coll. Int. CNRS (1974) 237, 59.

11. A. Starobinsky, JETP Lett. (1983) 37,66

12. R. Triay, Contemporary Math. (1997) 203,227

13. R. Triay, Int. Journ. Mod. Phys. A (2002) 17,29,4209 